\title{
Hábitos comportamentais em crianças e adolescentes com Paralisia Cerebral da cidade de Campina Grande, Paraíba
}

Behavioral habits in children and adolescents with Cerebral Palsy in Campina Grande, Brazil

Francisco Naldo Gomes Filho'; Rênnis Oliveira da Silva1; Mariana Marinho Davino de Medeiros²; Andreia Medeiros Rodrigues Cardoso3; Wilton Wilney Nascimento Padilha4; Alessandro Leite Cavalcanti ${ }^{5}$.

${ }^{1}$ Acadêmico de Odontologia da Universidade Federal da Paraíba, João Pessoa-PB-Brasil; 2Mestranda do Programa de Pós-Graduação em Clínica Odontológica da Faculdade de Odontologia de Piracicaba - Unicamp, Piracicaba-SP-Brasil;

${ }^{3}$ Professora Curso de Odontologia, Centro Universitário de João Pessoa, João Pessoa-PB-Brasil; ${ }^{4}$ Professor do Programa de Pós-graduação em Odontologia, Universidade Federal da Paraíba, João Pessoa-PB-Brasil;

${ }^{5}$ Professor do Curso de Odontologia, Universidade Estadual da Paraíba, Campina Grande-PBBrasil.

Andreia Medeiros Rodrigues Cardoso - Rua Comerciante Aristides Costa, n 140, Apto. 102, Jardim Cidade Universitária, João Pessoa, PB, Brasil. - CEP: 58052-240. Email: andreiamedeiros29@yahoo.com.br.

\section{RESUMO}

Introdução: Um dos principais fatores que comprometem a saúde bucal dos indivíduos com Paralisia Cerebral (PC) são os hábitos comportamentais, como higiene oral, dieta e hábitos deletérios. Objetivo: Identificar comportamentos relacionados à higiene oral, alimentação e hábitos deletérios em crianças e adolescentes com PC de Campina Grande, PB. Metodologia: Estudo transversal, de procedimento descritivo e técnica de observação direta. Amostra de 80 pacientes de 2 a 18 anos de idade com PC. Coleta de dados realizada em uma Associação para Excepcionais. Instrumento sobre dados socioeconômicos e hábitos comportamentais. Resultados: Os dados sociecoeconômicos mostraram que $52,5 \%$ das famílias tinha renda de até dois salários mínimos e $56,3 \%$ tinha baixa escolaridade. A dieta de $57,5 \%$ era de consistência sólida, $68,7 \%$ consumia mais de dois lanches ao longo do dia e $72,5 \%$ consumia algum medicamento. Quanto à higiene oral, 93,8\% utilizava escova e dentifrício, 81,3\% realizava a escovação duas vezes ou mais ao dia, 61,3\% dos cuidadores afirmou ter dificuldade em realizá-la e $57,5 \%$ foi ao dentista há mais de um ano. Sobre os hábitos deletérios, 9,4\% chupava o dedo, 13,2\% usava chupeta, 77,5\% apresentou hipotonia labial, 12,5\% interposição labial, 62,3\% interposição lingual e 73,8\% respiração bucal. Conclusão: A amostra realizava a higiene oral frequentemente. Entretanto, os responsáveis relataram dificuldade em realizar a escovação dental. A consistência da dieta desse grupo foi predominantemente sólida. Grande parte da amostra não realizava sucção de dedo e de chupeta. Porém, a maioria apresentou outros hábitos deletérios, como a respiração bucal, hipotonia labial e interposição lingual.

Descritores: Paralisia Cerebral. Comportamentos Relacionados com a Saúde. Criança. Higiene Bucal. Comportamento Alimentar. 


\begin{abstract}
Introduction: One of the main factors that compromise the oral health of individuals with Cerebral Palsy is behavioral habits such as oral hygiene, diet and deleterious habits. Objective: To identify behaviors related to oral hygiene, eating and deleterious habits in children and adolescents with CP of Campina Grande, PB. Methodology: Crosssectional study, descriptive procedure and direct observation technique. Sample of 80 patients from 2 to 18 years of age with CP. Collection of data held in an Association for Exceptional. Instrument on socioeconomic data and behavioral habits. Results: The socioeconomic data showed that $52.5 \%$ of families had income of up to two minimum wages and $56.3 \%$ had low level of education. The diet of $57.5 \%$ had a solid consistency, $68.7 \%$ consumed more than two snacks throughout the day and $72.5 \%$ consumed some medication. Regarding oral hygiene, $93.8 \%$ used toothbrush and toothpaste, $81.3 \%$ performed brushing twice or more daily, $61.3 \%$ of caregivers reported having difficulty performing it and $57.5 \%$ went to the dentist there more than a year. Regarding the deleterious habits, $9.4 \%$ sucked the finger, $13.2 \%$ used pacifiers, $77.5 \%$ presented lip hypotonia, $12.5 \%$ lip interposition, $62.3 \%$ lingual interposition and $73.8 \%$ mouth breathing. Conclusion: The sample performed oral hygiene frequently. However, those responsible reported difficulty in performing dental brushing. The consistency of the diet of this group was predominantly solid. Much of the sample did not perform finger and pacifier suction. However, most presented other deleterious habits, such as mouth breathing, lip hypotonia and lingual interposition.
\end{abstract}

Keywords: Cerebral Palsy. Health Behavior. Child. Oral Hygiene. Feeding Behavior.

\title{
Introdução
}

O número de pessoas com alguma forma de deficiência foi estimado em cerca de 45 milhões no Brasil ${ }^{1}$. Dentre as incapacidades físicas e mentais, está a Paralisia Cerebral (PC), a qual é considerada a principal condição incapacitante na infância ${ }^{2}$. Esta condição apresenta prevalência de sete para cada 1.000 nascidos vivos, de acordo com o Departamento de Neurologia Infantil da Universidade de São Paulo.

A PC é definida como uma desordem motora crônica e não progressiva, decorrente de danos cerebrais precoces nos períodos pré, peri ou pós-natal, quando o sistema nervoso da criança ainda está em desenvolvimento ${ }^{3}$. Esta condição gera uma debilitação na coordenação de ações musculares, com resultante incapacidade em manter posturas e realizar movimentos normais ${ }^{4}$. Está comumente associada à epilepsia, anormalidades da fala, aprendizado, audição, visão e retardo mental ${ }^{5}$.

Em virtude das limitações, as crianças com PC necessitam de apoio para realizar o autocuidado diário, o que está diretamente relacionado ao grau de dificuldade de aprendizagem e ao comprometimento neurológico que elas apresentam ${ }^{6}$. Portanto, a PC requer ajuda 
complementar dos pais ou responsáveis, a fim de manter uma boa saúde bucal, que consiste em uma das principais necessidades médicas dos pacientes com deficiência ${ }^{7}$.

As doenças orais em pacientes com PC são clinicamente semelhantes aos encontrados na população em geral, mas desenvolvem-se com maior frequência e gravidade em comparação aos pacientes sem comprometimento neurológico ${ }^{8}$. Este fato explica-se devido à comportamentos que envolvem: alta ingestão de carboidratos pastosos, o uso de medicamentos com açúcar, a dificuldade em realizar a higiene bucal destes pacientes, hipotonia e outros hábitos deletérios ${ }^{3}$. Além disso, os agravos bucais que surgem podem prejudicar a nutrição, digestão, mastigação dos alimentos e fonação ${ }^{9}$, o que compromete seu processo de reabilitação, bem como, o seu bemestar $^{10}$.

Neste contexto, os principais fatores que comprometem a saúde bucal deste grupo são os hábitos comportamentais, assim como o conhecimento dos cuidadores acerca da saúde bucal, uma vez que os indivíduos com PC são altamente dependentes para realização de tarefas comuns ${ }^{2}$. Diante do exposto, o objetivo deste estudo foi identificar comportamentos, relacionados à higiene oral, alimentação e hábitos deletérios, em crianças com PC da cidade de Campina Grande, estado da Paraíba, Brasil.

\section{Metodologia}

Após aprovação do Comitê de Ética em Pesquisa (CEP) da Universidade Estadual da Paraíba (CAAE 20215413.4.0000.5187), foi realizado um estudo transversal, com abordagem quantitativa, procedimentos descritivos, método indutivo e técnica de observação direta intensiva e extensiva ${ }^{11}$.

Este estudo foi desenvolvido na Associação de Pais e Amigos de Excepcionais (APAE) da cidade de Campina Grande, localizada na mesorregião do Agreste do estado da Paraíba, Brasil. A APAE se constitui em uma instituição que oferta atividades terapêuticas, educativas e de integração social a pessoas com PC.

A população do estudo compreendeu todas as 97 crianças e adolescentes com PC participantes da APAE, com idades entre dois e 18 anos, e seus respectivos cuidadores. Considerou-se como perda a recusa em participar do estudo e o não comparecimento para realização do exame clínico, após três tentativas sucessivas. Todos os participantes/responsáveis assinaram o Termo de Consentimento Livre e Esclarecido (TCLE).

Foram considerados cuidadores, os indivíduos com idade igual ou superior a 18 anos, responsáveis pela tomada de decisões e realização das atividades de vida diária das pessoas com PC. 
A coleta de dados foi realizada na APAE, no período compreendido entre outubro de 2013 e março de 2014, por uma única examinadora e um anotador. Inicialmente, dados clínicos com informações referentes à localização da PC e ao tipo de disfunção neuromuscular foram coletados nos prontuários. Em seguida, em uma sala de atendimento da APAE, uma ficha clínica foi preenchida face a face com o cuidador. O instrumento utilizado continha informações sobre dados socioeconômicos (sexo e idade da criança, anos de escolaridade do cuidador, renda familiar mensal classificada com base no salário mínimo do Brasil), e comportamentais: higiene oral (frequência da escovação, instrumentos utilizados, momento da escovação, dificuldades e acesso ao dentista), alimentação (instrumento utilizado, consistência da dieta e frequência de lanches) e hábitos deletérios (sucção de dedo e sucção de chupeta).

Foi realizado o exame clínico geral, com o paciente sentado na sua própria cadeira de rodas ou em cadeira tradicional, após escovação dentária, sob supervisão ${ }^{16}$. Observou-se o tipo de respiração, habilidade de comunicação e presença de hipotonia labial ${ }^{10}$.

Os dados coletados foram tabulados e analisados no programa Statistical Package for Social Sciences (SPSS para Windows, versão 20.0, SPSS Inc, Chicago, EUA).

\section{Resultados}

Foram examinadas 80 crianças e adolescentes, sendo 42 (52,5\%) do sexo masculino e 38 $(47,5 \%)$ do feminino. Dos 17 pacientes não avaliados, três se recusaram a participar do estudo e 14 não compareceram ao exame clínico.

Em relação à amostra, a faixa etária mais prevalente foi de sete a 11 anos (46,3\%), portadores de tetraparesia $(56,2 \%)$ e disfunção neuromuscular do tipo espástica $(75,0 \%)$.

Analisando-se os dados socioeconômicos, a renda familiar mais comum (52,5\%) foi de até dois salários mínimos, sendo um salário mínimo correspondente à $\mathrm{R} \$ 724,00$ no ano da pesquisa [12], a escolaridade predominante dos responsáveis (56,3\%) foi o ensino fundamental completo, e a maioria $(96,3 \%)$ não possuía formação acadêmica.

Segundo a Tabela 1, a alimentação da maioria $(57,5 \%)$ das crianças e adolescentes com PC possuia consistência sólida, e 22,5\% utilizava mamadeira como um dos instrumentos de alimentação. A Tabela 2 mostra que 68,7\% consumia lanches, além do café da manhã, almoço e jantar, duas vezes ou mais ao longo do dia. 52,5\% dos resposáveis afirmou que recebeu orientação profissional na elaboração da dieta dos filhos. Além disso, 72,5\% consumia algum medicamento, sendo $77,6 \%$ do tipo anticonvulsivante, como mostra a Tabela 3. 


\begin{tabular}{lcc}
\hline \multicolumn{2}{c}{$\begin{array}{c}\text { Tabela 1 - Distribuição da consistência da alimentação das crianças com } \\
\text { Paralisia Cerebral, Campina Grande, 2014. }\end{array}$} \\
\hline \multicolumn{1}{c}{ Consistência } & Frequência & Percentual \\
Sólida & 46 & 57,5 \\
Semissólida & 19 & 23,8 \\
Líquida & 15 & 18,8 \\
Total & $\mathbf{8 0}$ & $\mathbf{1 0 0 , 0}$
\end{tabular}

\begin{tabular}{|c|c|c|}
\hline Consistência & Frequência & Percentual \\
\hline 0 & 1 & 1,3 \\
\hline 1 & 24 & 30,0 \\
\hline 2 & 34 & 42,5 \\
\hline 3 & 17 & 21,3 \\
\hline 4 & 3 & 3,8 \\
\hline 5 & 1 & 1,3 \\
\hline Total & 80 & 100,0 \\
\hline
\end{tabular}

\section{Tabela 3 - Distribuição do tipo de medicamento consumido pelas crianças com Paralisia Cerebral, Campina Grande, 2014.}

\begin{tabular}{lcc}
\hline \multicolumn{1}{c}{ Medicamento } & Frequência & Percentual \\
Anticonvulsivante & 45 & 78,9 \\
Calmante & 10 & 17,5 \\
Anti-histamínico & 1 & 1,8 \\
Antiespasmódico & 1 & 1,8 \\
Total & $\mathbf{5 7}$ & $\mathbf{1 0 0 , 0}$
\end{tabular}

Quanto aos hábitos de higiene oral deste grupo, 93,8\% utilizava escova e dentifrício, como mostra a Tabela 4, 81,3\% realizava a escovação duas vezes ou mais ao dia, e $35,4 \%$ escovava os dentes antes do café, após o almoço e antes de dormir. 55,0\% dos cuidadores receberam orientação de profissionais da saúde para a higiene oral das crianças e adolescentes com PC, mas $61,3 \%$ afirmou que tinha dificuldade em realizar a higiene, sendo a presença do reflexo de mordida a dificuldade predominante $(37,7 \%)$, como mostra a Tabela 5. 


\begin{tabular}{lcc}
\hline $\begin{array}{c}\text { Tabela } \\
\text { 4 - Distribuição dos instrumentos utilizados para higiene oral das } \\
\text { crianças com Paralisia Cerebral, Campina Grande, }\end{array}$ \\
\hline \multicolumn{1}{c}{ Instrumentos de Higiene } & Frequência & Percentual \\
Escova & 1 & 1,3 \\
Escova e dentifrício & 75 & 93,8 \\
Gaze & 3 & 3,8 \\
Cotonete & 1 & 1,3 \\
Total & $\mathbf{8 0}$ & $\mathbf{1 0 0 , 0}$ \\
& & \\
\hline Tabela 5 - Dificuldade relatadas pelo cuidador para realizar a higiene oral \\
$\quad$ das crianças com Paralisia Cerebral, Campina Grande, 2014. \\
\hline \multicolumn{1}{c}{ Dificuldade relatada } & Frequência & Percentual \\
Presença de movimentos involuntários & 9 & 17,0 \\
Mordidas & 2 & 3,8 \\
Falta de colaboração & 12 & 22,6 \\
Engole o dentifrício & 6 & 11,3 \\
Presença de reflexo de mordida & 20 & 37,7 \\
Não se aplica & 4 & 7,5 \\
Total & $\mathbf{5 3}$ & $\mathbf{1 0 0 , 0}$ \\
&
\end{tabular}

Segundo a Tabela $6,57,5 \%$ foi ao dentista há mais de 12 meses. A Tabela 7 mostra que a principal dificuldade que impede o acesso ao serviço odontológico é a baixa oferta de dentista $(79,2 \%)$.

\begin{tabular}{|c|c|c|}
\hline $\mathrm{N}^{0}$ de consultas & Frequência & Percentual \\
\hline Nenhuma vez & 46 & 57,5 \\
\hline Uma vez & 14 & 17,5 \\
\hline Duas vezes & 10 & 12,5 \\
\hline Três vezes & 3 & 3,8 \\
\hline Mais de três vezes & 7 & 8,8 \\
\hline Total & 80 & 100,0 \\
\hline
\end{tabular}

Em relação aos hábitos deletérios, 9,4\% realizava sucção do dedo (Tabela 8), 13,2\% fazia uso de chupeta (Tabela 9), 77,5\% apresentou hipotonia labial, 12,5\% interposição labial, 62,3\% interposição lingual e 73,8\% respiração bucal, como mostra a Tabela 8. 


\begin{tabular}{|c|c|c|c|}
\hline \multicolumn{2}{|c|}{ Dificuldade } & Frequência & Percentual \\
\hline \multicolumn{2}{|c|}{ Baixa oferta de dentista } & 38 & 79,2 \\
\hline \multicolumn{2}{|c|}{ Demora no agendamento } & 8 & 16,7 \\
\hline \multicolumn{2}{|c|}{$\begin{array}{l}\text { Indisponibilidade de realizar o procedimento } \\
\text { com anestesia geral }\end{array}$} & 2 & 4,2 \\
\hline \multicolumn{2}{|c|}{ Total } & 48 & 100,0 \\
\hline \multicolumn{4}{|c|}{$\begin{array}{l}\text { Tabela } 8 \text { - Frequência das crianças com PC que realizava sucção do dedo, } \\
\text { Campina Grande, } 2014 .\end{array}$} \\
\hline Resposta & Frequência & \multicolumn{2}{|c|}{ Percentual } \\
\hline Sim & 5 & \multicolumn{2}{|c|}{9,4} \\
\hline Não & 48 & \multicolumn{2}{|c|}{90,6} \\
\hline Total & 53 & \multicolumn{2}{|c|}{100,0} \\
\hline \multicolumn{4}{|c|}{$\begin{array}{l}\text { Tabela } 9 \text { - Distribuição do tipo de respiração das crianças com PC, } \\
\text { Campina Grande, } 2014 .\end{array}$} \\
\hline Respiração & Frequência & \multicolumn{2}{|c|}{ Percentual } \\
\hline Nasal & 21 & \multicolumn{2}{|c|}{26,3} \\
\hline Bucal & 59 & \multicolumn{2}{|c|}{73,8} \\
\hline Total & 80 & \multicolumn{2}{|c|}{100,0} \\
\hline
\end{tabular}

\section{Discussão}

Neste estudo, a maior parte dos responsáveis apresentou baixo nível de escolaridade e baixa renda, com a maioria $(52,5 \%)$ das famílias vivendo com até dois salários mínimos. Estudos de outras regiões do Brasil demonstraram resultados semelhante ${ }^{4,8}$. Possivelmente, isso se explica pelo fato de os responsáveis pelas crianças com PC, as quais possuem alta dependência, não exercerem trabalho remunerado para cuidarem delas, não podendo, assim, cooperar financeiramente com a renda familiar. De acordo com a literatura, o baixo nível socioeconômico é um dos fatores que dificulta o acesso ao material de higiene oral e ao tratamento odontológico, relacionando-se a baixa frequência de consulta odontológica relatados neste trabalho ${ }^{4,13}$.

Em contrapartida, foi frequente a utilização de escova e dentifrício $(93,8 \%)$ e a realização da escovação dentária, que acontecia duas vezes ou mais ao dia na maioria do casos (81,3\%), sendo considerados bons hábitos de higiene oral ${ }^{2,14}$. Entretanto, a maioria dos cuidadores afirmou encontrar dificuldades para realizar a higiene oral das crianças e adolescentes com PC $(61,3 \%)$, 
resultado similar encontrado na literatura ${ }^{15}$. Isso se deve à presença de movimentos involuntários, reflexos orais patológicos, deglutição de dentifrício, reflexo de mordida, espasticidade dos músculos da mastigação, medo, falta de cooperação e limitação de abertura bucal ${ }^{16}$.

Outrossim, foi relatado pelos responsáveis que há uma baixa oferta de dentistas, sendo uma das dificuldades de acesso ao serviço odontológico, podendo ocasionar, portanto, a falta de informação e instrução qualificadas, acerca dos cuidados específicos com a saúde bucal das crianças com PC, acarretando em uma higiene oral não eficiente ${ }^{4}$.

A predominância $(57,5 \%)$ da alimentação com consistência sólida nas refeições dos indivíduos com PC, explica a baixa frequência (22,5\%) do uso da mamadeira e demonstra uma possível evolução na função alimentar motivados pela fonoterapia, a qual é um dos tratamentos, ofertado pela APAE, para Paralisia Cerebral que visa uma reabilitação da disfagia orofaríngea acarretadas por essa condição ${ }^{17}$. Além disso, a alta frequência da realização diária de lanches encontrada neste estudo, pode ser explicada pela intenção do cuidador em aumentar a ingestão de carboidratos, de forma a evitar desnutrição das crianças com $\mathrm{PC}^{7}$. Contudo, esses carboidratos são, geralmente, de consistência líquida ou pastosa por serem facilmente consumidos e digeridos ${ }^{11}$, sendo, assim, um fator também responsável pelo favorecendo do desenvolvimento de cárie dentária e doença periodontal, associado à redução da função de auto limpeza da cavidade bucal, devido à presença de condições limitantes inerentes à Paralisia Cerebral ${ }^{18}$.

O consumo frequente $(78,9 \%)$ de anticonvulsivantes entre os indivíduos com PC demonstra o potencial risco de aparecimento de agravos bucais, como a cárie dentária e alterações periodontais, tendo em vista que esses medicamentos são caracterizados por alto conteúdo de açúcar, baixo $\mathrm{pH}$ e elevada viscosidade, favorecendo, portanto, a proliferação de biofilmes cariogênicos e a desmineralização do esmalte dentário, propiciando o desenvolvimento do processo da cárie e da gengivite ${ }^{8,19}$. Apesar disso, não se deve retirar o seu uso, pois são essenciais para a boa saúde das crianças com Paralisia Cerebral ${ }^{19}$.

O hábito de succionar o dedo esteve presente em 9,4\% e usar chupeta em 13,2\% das crianças, sendo uma frequência relativamente baixa se comparada a outros estudos ${ }^{20}$. Isso pode se explicar pelo fato de os responsáveis terem impedido os hábitos deletérios citados, após conhecimento dos seus malefícios para a criança, como a má oclusão ${ }^{20}$.Entretanto, grande parte da amostra apresentou hipotonia labial (77,5\%), interposição lingual $(62,3 \%)$ e respiração bucal (73,8\%), o que demonstra os efeitos da disfunção muscular acarretada pelo comprometimento do tônus muscular advindo da Paralisia Cerebral ${ }^{16}$.

Este estudo apresenta limitações que afetam a interpretação dos resultados. Por ser um estudo transversal, não é possível estabelecer relações de causa e efeito, demonstrando exclusivamente o perfil encontrado neste grupo de forma descritiva. Reforça-se a realização de 
mais investigações que busquem verificar os hábitos comportamentais dos pacientes com PC relacionados com agravos bucais, principalmente, estudos longitudinais, com maiores tamanhos de amostras e critérios de seleção semelhantes aos estudos existentes, para que comparações possam ser realizadas.

No entanto, há uma carência de estudos relacionados aos hábitos comportamentais em pacientes com PC, destacando-se, assim, a importância da investigação realizada neste estudo, tendo em vista que esses hábitos influenciam na saúde bucal, a qual é um dos principais componentes da saúde geral e da qualidade de vida de uma pessoa².

Diante do exposto, recomenda-se o desenvolvimento de estratégias de promoção em saúde bucal para crianças com Paralisia Cerebral, a fim de instruir os cuidadores acerca da adequada higiene oral, enfatizando os cuidados específicos e os agravos que podem surgir devido à negligência. Deve-se estimular o tratamento fonoaudiólogo, com o intuito de minimizar os efeitos dos hábitos deletérios inerentes à PC, e buscar a evolução alimentar, como foi constatada em parte da amostra. Além disso, sugere-se que a composição dos medicamentos consumidos por este grupo seja modificada, de modo a minimizar seus efeitos colaterais cariogênicos. Ademais, são necessárias ações que visem orientar e motivar os responsáveis em relação à manutenção da saúde dos filhos com PC, tendo em vista as dificuldades e limitações que esta condição acarreta.

\section{Conclusão}

Os resultados deste estudo, na cidade de Campina Grande, indicaram que, apesar da baixa escolaridade e baixa renda dos responsáveis, as crianças com Paralisia Cerebral realizavam a higiene oral de forma frequente. Entretanto, os responsáveis relataram dificuldade em realizar a escovação dental. Além disso, foi constada a baixa oferta de dentista, o que compromete a propagação de informação qualificada acerca da saúde bucal. Verificou-se que a consistência da dieta desse grupo foi predominantemente sólida, demonstrando uma evolução alimentar de uma parcela das crianças. Houve um grande consumo de anticonvulsivantes pelas crianças com PC. A maior parte da amostra não realizava sucção de dedo e de chupeta; porém, a maioria apresentou outras complicações, como a respiração bucal, hipotonia labial e interposição lingual.

Estes dados são de potencial valor para melhorar as atividades de promoção da saúde bucal voltadas a pacientes com PC, com o intuito de orientar os responsáveis acerca dos cuidados específicos que devem ser tomados desde a primeira infância, devido às limitações presentes nesse grupo, a fim de que as crianças com Paralisia Cerebral tenham um desenvolvimento saudável. 


\section{Referências}

1. Instituto Brasileiro de Geografia e Estatística. Censo Demográfico 2010: características gerais da população, religião e deficiência. Banco de Dados Agregados. 2012.

2. Du RY, McGrath CP, Yiu CKY, King NM. Oral health behaviors of preschool children with cerebral palsy: a case-control community-based study. Spec Care Dent.2014;34(6):298-302.

3. Carvalho RB, Mendes RF, Prado Jr RR, Neto JMM. Oral health and oral motor function in children with cerebral palsy. Spec Care Dent. Wiley. 2011;31(2):58-62.

4. Lemos ACO, Katz CRT. Condições de saúde bucal e acesso ao tratamento odontológico de pacientes com paralisia cerebral atendidos em um centro de referência do Nordeste - Brasil. Rev CEFAC. 5 de junho de 2012;14(5):861-871.

5. Oliveira ET, Carvalho AC, Matinelli J, Thedei-Júnior G, Lepri CP, Menezes-Oliveira MAH. Avaliação da condição bucal de pacientes portadores de paralisia cerebral na cidade de Uberaba. Arq Cent Estud Curso Odontol Univ Fed Minas Gerais. 2016;52(2):88-93.

6. Ketelaar M, Gorter JW, Westers P, Hanna S, Verhoef M. Developmental Trajectories of Mobility and Self-Care Capabilities in Young Children with Cerebral Palsy. J Pediatr. 2014;164(4):769-774.e2.

7. Liu Z, Yu D, Luo W, Yang J, Lu J, Gao S, et al. Impact of oral health behaviors on dental caries in children with intellectual disabilities in Guangzhou, China. Int J Environ Res Public Health. 2014;11(10):1015-1027.

8. Guerreiro PO, Garcias G de L. Diagnóstico das condições de saúde bucal em portadores de paralisia cerebral do município de Pelotas, Rio Grande do Sul, Brasil. Cien Saude Colet. 2009;14(5):1939-1946.

9. Anders PL, Davis EL. Oral health of patients with intellectual disabilities: A systematic review. Spec Care Dent. 2010;30(3):110-117.

10. Cardoso AMR. Cárie Dentária e Alterações Periodontais em Crianças e Adolescentes Brasileiros com Paralisia Cerebral. Universidade Estadual da Paraíba; 25 de julho de 2014.

11. Lakatos EM, Marconi M de A. Fundamentos da metodologia científica. $6^{\circ}$ ed. São Paulo: Atlas; 2009.

12. BRASIL. Valor do Salário Mínimo. Decreto $n^{\circ} 8166$ 2013, de 23 de dezembro de 2013. Valor do Salário Mínimo. Presidência da República, Casa Civil. 23 dez 2013.

13. Santos MTBR, Masiero D, Simionato MRL. Risk factors for dental caries in children with cerebral palsy. Spec Care Dentist. 2002;22(3):103-107.

14. Levine R, Stillman-Lowe C. The scientific basis of dental health education. Community Dent Health. 2002;19(2):127. 
15. Minihan PM, Morgan JP, Park A, Yantsides KE, Nobles CJ, Finkelman MD, et al. At-home oral care for adults with developmental disabilities. J Am Dent Assoc. 2014;145(10):10181025.

16. Cardoso A, Gomes L, Silva C, Soares R, de Abreu M, Padilha W, et al. Dental Caries and Periodontal Disease in Brazilian Children and Adolescents with Cerebral Palsy. Int J Environ Res Public Health. 2014;12(1):335-353.

17. Menezes E da C, Santos FAH, Alves FL, Menezes E da C, Santos FAH, Alves FL. Cerebral palsy dysphagia: a systematic review. Rev CEFAC. 2017;19(4):565-574.

18. Guaré RO, Fernandes DCGN, Costa MB da, Santos MTBR. Saúde bucal e qualidade de vida em crianças com paralisia cerebral. Rev Bras Pesqui em Saúde. 2015;16(3):7-13.

19. Alves VF, Cardoso AMR, Cavalcanti YW, Padilha WWN. Efeito sobre a morfologia do esmalte dental e análise físico-química de medicamentos utilizados por pacientes pediátricos com paralisia cerebral. Rev Odontol da UNESP. 2016; 45(4):201-205.

20. Castilho LS de, Abreu MHNG, Ribeiro LVL, Silva MES e, Resende VLS. Perfil dos pacientes com deficiências de desenvolvimento sob atendimento odontológico em um projeto de extensão intersetorial. Arq em Odontol. 2017;53(1). 